\title{
A class of weighted Hardy inequalities and applications to evolution problems
}

\author{
Anna Canale $^{1}$ (D) . Francesco Pappalardo ${ }^{2}$ (D) Ciro Tarantino $^{3}$ (D)
}

Received: 4 April 2019 / Accepted: 21 September 2019 / Published online: 3 October 2019

(c) Fondazione Annali di Matematica Pura ed Applicata and Springer-Verlag GmbH Germany, part of Springer Nature 2019

\section{Abstract}

We state the following weighted Hardy inequality:

$$
c_{o, \mu} \int_{\mathbb{R}^{N}} \frac{\varphi^{2}}{|x|^{2}} \mathrm{~d} \mu \leq \int_{\mathbb{R}^{N}}|\nabla \varphi|^{2} \mathrm{~d} \mu+K \int_{\mathbb{R}^{N}} \varphi^{2} \mathrm{~d} \mu \quad \forall \varphi \in H_{\mu}^{1},
$$

in the context of the study of the Kolmogorov operators:

$$
L u=\Delta u+\frac{\nabla \mu}{\mu} \cdot \nabla u,
$$

perturbed by inverse square potentials and of the related evolution problems. The function $\mu$ in the drift term is a probability density on $\mathbb{R}^{N}$. We prove the optimality of the constant $c_{o, \mu}$ and state existence and nonexistence results following the Cabré-Martel's approach (Cabré and Martel in C R Acad Sci Paris 329 (11): 973-978, 1999) extended to Kolmogorov operators.

Keywords Weighted Hardy inequality · Optimal constant · Kolmogorov operators · Singular potentials

Mathematics Subject Classification $35 \mathrm{~K} 15 \cdot 35 \mathrm{~K} 65 \cdot 35 \mathrm{~B} 25 \cdot 34 \mathrm{G} 10 \cdot 47 \mathrm{D} 03$

The first two authors are members of the Gruppo Nazionale per l'Analisi Matematica, la Probabilitá e le loro Applicazioni (GNAMPA) of the Istituto Nazionale di Alta Matematica (INdAM).

\section{Anna Canale}

acanale@unisa.it

Francesco Pappalardo

francesco.pappalardo@unina.it

Ciro Tarantino

ctarant@unina.it

1 Dipartimento di Ingegneria dell'Informazione ed Elettrica e Matematica Applicata, Universitá degli Studi di Salerno, Via Giovanni Paolo II, 132, 84084 Fisciano, SA, Italy

2 Dipartimento di Matematica e Applicazioni "Renato Caccioppoli", Universitá degli Studi di Napoli Federico II, Complesso Universitario Monte S. Angelo, Via Cintia, 80126 Naples, Italy

3 Dipartimento di Scienze Economiche e Statistiche, Universitá degli Studi di Napoli Federico II, Complesso Universitario Monte S. Angelo, Via Cintia, 80126 Naples, Italy 


\section{Introduction}

This paper on weighted Hardy inequalities fits in the framework of the study of Kolmogorov operators on smooth functions:

$$
L u=\Delta u+\frac{\nabla \mu}{\mu} \cdot \nabla u,
$$

where $\mu$ is a probability density on $\mathbb{R}^{N}$, and of the related evolution problems:

$$
(P) \quad\left\{\begin{array}{l}
\partial_{t} u(x, t)=L u(x, t)+V(x) u(x, t), \quad x \in \mathbb{R}^{N}, \quad t>0, \\
u(\cdot, 0)=u_{0} \geq 0 \in L_{\mu}^{2} .
\end{array}\right.
$$

The operator $L$ in $(P)$ is perturbed by the singular potential $V(x)=\frac{c}{|x|^{2}}, c>0$, and $L_{\mu}^{2}:=L\left(\mathbb{R}^{N}, \mathrm{~d} \mu\right)$, with $\mathrm{d} \mu(x)=\mu(x) \mathrm{d} x$.

The interest in inverse square potentials of type $V \sim \frac{c}{|x|^{2}}$ relies in their criticality: the strong maximum principle and Gaussian bounds fail (see [2]). Furthermore, interest in singular potentials is due to the applications to many fields, for example in many physical contexts as molecular physics [23], quantum cosmology (see, e.g., [5]), quantum mechanics [4] and combustion models [19].

The operator $\Delta+V, V(x)=\frac{c}{|x|^{2}}$, has the same homogeneity as the Laplacian and does not belong to the Kato class, then, $V$ cannot be regarded as a lower order perturbation term.

A remarkable result stated in 1984 by P. Baras and J. A. Goldstein in [3] shows that the evolution problem $(P)$ with $L=\Delta$ admits a unique positive solution if $c \leq c_{o}=\left(\frac{N-2}{2}\right)^{2}$ and no positive solutions exist if $c>c_{o}$. When it exists, the solution is exponentially bounded, on the contrary, if $c>c_{o}$, there is the so-called instantaneous blow-up phenomenon.

In order to extend these results to Kolmogorov operators, the technique must be different.

A result analogous to that stated in [3] has been obtained in 1999 by X. Cabré and Y. Martel [8] for more general potentials $0 \leq V \in L_{\text {loc }}^{1}\left(\mathbb{R}^{N}\right)$ with a different approach.

To state the existence and nonexistence results, we follow the Cabré-Martel's approach. We use the relation between the weak solution of $(P)$ and the bottom of the spectrum of the operator $-(L+V)$ :

$$
\lambda_{1}(L+V):=\inf _{\varphi \in H_{\mu}^{1} \backslash\{0\}}\left(\frac{\int_{\mathbb{R}^{N}}|\nabla \varphi|^{2} \mathrm{~d} \mu-\int_{\mathbb{R}^{N}} V \varphi^{2} \mathrm{~d} \mu}{\int_{\mathbb{R}^{N}} \varphi^{2} \mathrm{~d} \mu}\right)
$$

where $H_{\mu}^{1}$ is the suitable weighted Sobolev space.

When $\mu=1$, Cabré and Martel showed that the boundedness of $\lambda_{1}(\Delta+V), 0 \leq V \in$ $L_{\text {loc }}^{1}\left(\mathbb{R}^{N}\right)$, is a necessary and sufficient condition for the existence of positive exponentially bounded in time solutions to the associated initial value problem. Later in [9,20], similar results have been extended to Kolmogorov operators. The proof uses some properties of the operator $L$ and of its corresponding semigroup in $L_{\mu}^{2}\left(\mathbb{R}^{N}\right)$.

For Ornstein-Uhlenbeck-type operators, $L u=\Delta u-\sum_{i=1}^{n} A\left(x-a_{i}\right) \cdot \nabla u, a_{i} \in \mathbb{R}^{N}$, $i=1, \ldots, n$, perturbed by multipolar inverse square potentials, weighted multipolar Hardy inequalities and related existence and nonexistence results were stated in [11]. In such a case, the invariant measure for these operators is $\mathrm{d} \mu=\mu_{A}(x) \mathrm{d} x=K e^{-\frac{1}{2} \sum_{i=1}^{n}\left\langle A\left(x-a_{i}\right), x-a_{i}\right\rangle} \mathrm{d} x$.

There is a close relation between the estimate of the bottom of the spectrum $\lambda_{1}(L+V)$ and the weighted Hardy inequality with $V(x)=\frac{c}{|x|^{2}}, c \leq c_{o, \mu}$,

$$
\int_{\mathbb{R}^{N}} V \varphi^{2} \mathrm{~d} \mu \leq \int_{\mathbb{R}^{N}}|\nabla \varphi|^{2} \mathrm{~d} \mu+K \int_{\mathbb{R}^{N}} \varphi^{2} \mathrm{~d} \mu \quad \forall \varphi \in H_{\mu}^{1}, \quad K>0
$$


with the best possible constant $c_{o, \mu}$.

In particular, the existence of positive solutions to $(P)$ is related to the Hardy inequality (1) and the nonexistence is due to the optimality of the constant $c_{o, \mu}$.

The main results in the paper are, in Sect. 2, the weighted Hardy inequality (1) with measures which satisfy fairly general conditions and the optimality of the constant $c_{o, \mu}$ in Sect. 3.

The proof of the weighted Hardy inequality is different from the others in the literature. It is based on the introduction of a suitable $C^{\infty}$ function, and it can be used to prove inequality (1) with $0 \leq V \in L_{\text {loc }}^{1}\left(\mathbb{R}^{N}\right)$ of a more general type, in other words Hardy type inequalities.

In [9], the authors state a weighted Hardy inequality using a different approach and improved Hardy inequalities. This requires suitable conditions on $\mu$. Our technique, with different assumptions on $\mu$, allows us to achieve the best constant (cf. [9, Theorem 3.3]) for a wide class of functions $\mu$. To state the optimality of the constant in the estimate, we need further assumptions on $\mu$ as usually it is done. We find a suitable function $\varphi$ for which the inequality (1) does not hold if $c>c_{o, \mu}$, and this is a crucial point in the proof. The way to estimate the bottom of the spectrum is close to the one used in [9]. We remark that the inequality obtained under our hypotheses applies in the context of weighted multipolar Hardy inequalities stated in the forthcoming paper [12].

Finally, we state an existence and nonexistence result in Sect. 4 following the CabréMartel's approach and using some results stated in $[9,20]$ when the function $\mu$ belongs to $C_{\text {loc }}^{1, \lambda}\left(\mathbb{R}^{N}\right)$ or belongs to $C_{\text {loc }}^{1, \lambda}\left(\mathbb{R}^{N} \backslash\{0\}\right)$, for some $\lambda \in(0,1)$.

Some classes of functions $\mu$ satisfying the hypotheses of the main Theorems are given in Sect. 2.

\section{Weighted Hardy inequalities}

Let $\mu$ be a weight function in $\mathbb{R}^{N}$. We define the weighted Sobolev space $H_{\mu}^{1}=$ $H^{1}\left(\mathbb{R}^{N}, \mu(x) \mathrm{d} x\right)$ as the space of functions in $L_{\mu}^{2}:=L^{2}\left(\mathbb{R}^{N}, \mu(x) \mathrm{d} x\right)$ whose weak derivatives belong to $\left(L_{\mu}^{2}\right)^{N}$.

As first step, we consider the following conditions on $\mu$ which we need to state a preliminary weighted Hardy inequality:

$\left(H_{1}\right) \quad \mu \geq 0, \mu \in L_{\mathrm{loc}}^{1}\left(\mathbb{R}^{N}\right)$;

$\left(H_{2}\right) \quad \nabla \mu \in L_{\text {loc }}^{1}\left(\mathbb{R}^{N}\right)$;

$\left(H_{3}\right) \quad$ there exist constants $k_{1}, k_{2} \in \mathbb{R}, k_{2}>2-N$, such that if

$$
f_{\varepsilon}=\left(\varepsilon+|x|^{2}\right)^{\frac{\alpha}{2}}, \quad \alpha<0, \quad \varepsilon>0,
$$

it holds

$$
\frac{\nabla f_{\varepsilon}}{f_{\varepsilon}} \cdot \nabla \mu=\frac{\alpha x}{\varepsilon+|x|^{2}} \cdot \nabla \mu \leq\left(k_{1}+\frac{k_{2} \alpha}{\varepsilon+|x|^{2}}\right) \mu
$$

for any $\varepsilon>0$.

The condition $\left(H_{3}\right)$ contains the requirement that the scalar product $\alpha x \cdot \frac{\nabla \mu}{\mu}$ is bounded in $B_{R}, R>0$, while $\frac{\alpha x}{\varepsilon+|x|^{2}} \cdot \frac{\nabla \mu}{\mu}$ is bounded in $\mathbb{R}^{N} \backslash B_{R}$, where $B_{R}$ is a ball of radius $R$ centered in zero.

The reason we use the function $f_{\varepsilon}$, introduced in [17], will be clear in the proof of the weighted Hardy inequality which we will state below. Finally, we observe that we need the condition $k_{2}>2-N$ to apply Fatou's lemma in the proof of Theorem 1 . 
Theorem 1 Under conditions $\left(H_{1}-H_{3}\right)$, there exists a positive constant $c$ such that

$$
c \int_{\mathbb{R}^{N}} \frac{\varphi^{2}}{|x|^{2}} \mathrm{~d} \mu \leq \int_{\mathbb{R}^{N}}|\nabla \varphi|^{2} \mathrm{~d} \mu+k_{1} \int_{\mathbb{R}^{N}} \varphi^{2} \mathrm{~d} \mu,
$$

for any function $\varphi \in C_{c}^{\infty}\left(\mathbb{R}^{N}\right)$, where $c \in\left(0, c_{o}\left(N+k_{2}\right)\right]$ with $c_{o}\left(N+k_{2}\right)=\left(\frac{N+k_{2}-2}{2}\right)^{2}$.

Proof As first step, we start from the integral of the square of the gradient of the function $\varphi$. Then, we introduce $\psi=\frac{\varphi}{f_{\varepsilon}}$, with $f_{\varepsilon}$ defined in $\left(H_{3}\right)$, and integrate by parts taking in mind $\left(H_{1}\right)$ and $\left(H_{2}\right)$.

$$
\begin{aligned}
\int_{\mathbb{R}^{N}}|\nabla \varphi|^{2} \mathrm{~d} \mu= & \int_{\mathbb{R}^{N}}\left|\nabla\left(\psi f_{\varepsilon}\right)\right|^{2} \mathrm{~d} \mu \\
= & \int_{\mathbb{R}^{N}}\left|\nabla \psi f_{\varepsilon}+\nabla f_{\varepsilon} \psi\right|^{2} \mathrm{~d} \mu \\
= & \int_{\mathbb{R}^{N}}|\nabla \psi|^{2} f_{\varepsilon}^{2} \mathrm{~d} \mu+\int_{\mathbb{R}^{N}} \psi^{2}\left|\nabla f_{\varepsilon}\right|^{2} \mathrm{~d} \mu+2 \int_{\mathbb{R}^{N}} f_{\varepsilon} \psi \nabla \psi \cdot \nabla f_{\varepsilon} \mathrm{d} \mu \\
= & \int_{\mathbb{R}^{N}}|\nabla \psi|^{2} f_{\varepsilon}^{2} \mathrm{~d} \mu+\int_{\mathbb{R}^{N}} \psi^{2}\left|\nabla f_{\varepsilon}\right|^{2} \mathrm{~d} \mu \\
& -\int_{\mathbb{R}^{N}} \psi^{2}\left|\nabla f_{\varepsilon}\right|^{2} \mathrm{~d} \mu-\int_{\mathbb{R}^{N}} f_{\varepsilon}^{2} \psi^{2} \frac{\Delta f_{\varepsilon}}{f_{\varepsilon}} \mathrm{d} \mu-\int_{\mathbb{R}^{N}} f_{\varepsilon}^{2} \psi^{2} \frac{\nabla f_{\varepsilon}}{f_{\varepsilon}} \cdot \nabla \mu \mathrm{d} x .
\end{aligned}
$$

Observing that

$$
\Delta f_{\varepsilon}=\frac{\alpha(N-2+\alpha)|x|^{2}+\alpha \varepsilon N}{\left(\varepsilon+|x|^{2}\right)^{2-\frac{\alpha}{2}}}
$$

and using hypothesis $\left(H_{3}\right)$, we deduce that

$$
\begin{aligned}
\int_{\mathbb{R}^{N}}|\nabla \varphi|^{2} \mathrm{~d} \mu \geq & -\int_{\mathbb{R}^{N}} \frac{\Delta f_{\varepsilon}}{f_{\varepsilon}} \varphi^{2} \mathrm{~d} \mu-\int_{\mathbb{R}^{N}} \frac{\nabla f_{\varepsilon}}{f_{\varepsilon}} \cdot \nabla \mu \varphi^{2} \mathrm{~d} x \\
\geq & -\left[\alpha(N-2)+\alpha^{2}\right] \int_{\mathbb{R}^{N}} \frac{|x|^{2}}{\left(\varepsilon+|x|^{2}\right)^{2}} \varphi^{2} \mathrm{~d} \mu-\varepsilon \alpha N \int_{\mathbb{R}^{N}} \frac{\varphi^{2}}{\left(\varepsilon+|x|^{2}\right)^{2}} \mathrm{~d} \mu \\
& -k_{1} \int_{\mathbb{R}^{N}} \varphi^{2} \mathrm{~d} \mu-k_{2} \alpha \int_{\mathbb{R}^{N}} \frac{\varphi^{2}}{\varepsilon+|x|^{2}} \mathrm{~d} \mu \\
= & {\left[-\alpha\left(N-2+k_{2}\right)-\alpha^{2}\right] \int_{\mathbb{R}^{N}} \frac{|x|^{2}}{\left(\varepsilon+|x|^{2}\right)^{2}} \varphi^{2} \mathrm{~d} \mu } \\
& -\varepsilon \alpha\left(N+k_{2}\right) \int_{\mathbb{R}^{N}} \frac{\varphi^{2}}{\left(\varepsilon+|x|^{2}\right)^{2}} \mathrm{~d} \mu-k_{1} \int_{\mathbb{R}^{N}} \varphi^{2} \mathrm{~d} \mu .
\end{aligned}
$$

The constant $-\alpha\left(N-2+k_{2}\right)-\alpha^{2}$ is greater than zero for $-\left(N-2+k_{2}\right)<\alpha<0$ and $k_{2}>2-N$, so by Fatou's lemma, we state the following estimate letting $\varepsilon \rightarrow 0$ :

$$
\int_{\mathbb{R}^{N}}|\nabla \varphi|^{2} \mathrm{~d} \mu+k_{1} \int_{\mathbb{R}^{N}} \varphi^{2} \mathrm{~d} \mu \geq c \int_{\mathbb{R}^{N}} \frac{\varphi^{2}}{|x|^{2}} \mathrm{~d} \mu,
$$

where $c=-\alpha\left(N-2+k_{2}\right)-\alpha^{2}$. Finally, we observe that

$$
\max _{\alpha}\left[-\alpha\left(N+k_{2}-2\right)-\alpha^{2}\right]=\left(\frac{N+k_{2}-2}{2}\right)^{2}=: c_{o}\left(N+k_{2}\right),
$$


attained for $\alpha_{o}=-\frac{N+k_{2}-2}{2}$.

Remark 1 In an alternative way, we can define $f_{\varepsilon}$ in $\left(H_{3}\right)$ setting $\alpha=\alpha_{o}$ and get the estimate (2) with $c=c_{o}\left(N+k_{2}\right)$. Although the result goes in the same direction, in the proof we point out that $c_{o}\left(N+k_{2}\right)$ is the maximum value of the constant $c$.

Remark 2 In the case $\mu=1$, we obtain the classical Hardy inequality. We remark that if in the proof we introduce a function $f \in C^{\infty}\left(\mathbb{R}^{N}\right)$ in place of $f_{\varepsilon}$, the inequality (4) can be used to get Hardy type inequalities:

$$
\int_{\mathbb{R}^{N}} V \varphi^{2} \mathrm{~d} x \leq \int_{\mathbb{R}^{N}}|\nabla \varphi|^{2} \mathrm{~d} x
$$

where the potential $V=V(x) \in L_{\mathrm{loc}}^{1}\left(\mathbb{R}^{N}\right), V(x) \geq 0$, is such that

$$
-\frac{\Delta f}{f} \geq V \quad \forall x \in \mathbb{R}^{N} .
$$

Operators perturbed by potentials of a more general type, for which the generation of semigroups was stated, have been investigated, for example, in [13-15] when $\mu=1$ and in [10] in weighted spaces. For functions $\mu \neq 1$ such that $k_{2} \neq 0$, we have to modify the condition $\left(H_{3}\right)$ to get the Hardy type inequality (5) with respect to the measure $\mathrm{d} \mu$.

Now, we suppose that

( $\left.H_{4}\right) \quad \mu \geq 0, \sqrt{\mu} \in H_{\mathrm{loc}}^{1}\left(\mathbb{R}^{N}\right)$;

( $\left.H_{5}\right) \quad \mu^{-1} \in L_{\text {loc }}^{1}\left(\mathbb{R}^{N}\right)$.

Let us observe that in the hypotheses $\left(H_{4}-H_{5}\right)$, the space $C_{c}^{\infty}\left(\mathbb{R}^{N}\right)$ is dense in $H_{\mu}^{1}$, and $H_{\mu}^{1}$ is the completion of $C_{c}^{\infty}\left(\mathbb{R}^{N}\right)$ with respect to the Sobolev norm:

$$
\|\cdot\|_{H_{\mu}^{1}}^{2}:=\|\cdot\|_{L_{\mu}^{2}}^{2}+\|\nabla \cdot\|_{L_{\mu}^{2}}^{2}
$$

(see [25]). For some interesting papers on density of smooth functions in weighted Sobolev spaces and related questions, we refer, for example, to [6,7,16,18,21,22,26].

So, we can deduce the following result from Theorem 1 by density argument.

Theorem 2 Under conditions $\left(\mathrm{H}_{2}-\mathrm{H}_{5}\right)$, there exists a positive constant $c$ such that

$$
c \int_{\mathbb{R}^{N}} \frac{\varphi^{2}}{|x|^{2}} \mathrm{~d} \mu \leq \int_{\mathbb{R}^{N}}|\nabla \varphi|^{2} \mathrm{~d} \mu+k_{1} \int_{\mathbb{R}^{N}} \varphi^{2} \mathrm{~d} \mu,
$$

for any function $\varphi \in H_{\mu}^{1}$, where $c \in\left(0, c_{o}\left(N+k_{2}\right)\right]$ with $c_{o}\left(N+k_{2}\right)=\left(\frac{N+k_{2}-2}{2}\right)^{2}$.

We give some examples of functions $\mu$ which satisfy the hypotheses of Theorem 2.

We remark that, in the hypotheses $\mu=\mu(|x|) \in C^{1}$ for $|x| \in\left[r_{0},+\infty\left[, r_{0}>0\right.\right.$, a class of weight functions $\mu$ which satisfies $\left(H_{3}\right)$ is the following:

$$
\mu(x) \geq C e^{-\frac{k_{1}}{2|\alpha|}|x|^{2}}|x|^{k_{2}-\frac{k_{1}}{|\alpha|} \varepsilon}, \text { for }|x| \geq r_{0},
$$

where $C$ is a constant depending on $\mu\left(r_{0}\right)$ and $r_{0}$.

Indeed, in the case of radial functions, $\mu(x)=\mu(|x|)$, if we set $|x|=\rho$, the condition $\left(H_{3}\right)$ states that $\mu$ satisfies the following inequality:

$$
\frac{\alpha \rho}{\varepsilon+\rho^{2}} \mu^{\prime}(\rho) \leq\left(k_{1}+\frac{k_{2} \alpha}{\varepsilon+\rho^{2}}\right) \mu(\rho),
$$


which implies

$$
\mu^{\prime}(\rho) \geq a(\rho) \mu(\rho)
$$

where

$$
a(\rho)=\frac{k_{1}}{\alpha}\left(\frac{\varepsilon+\rho^{2}}{\rho}\right)+\frac{k_{2}}{\rho} .
$$

Integrating in $\left[r_{0}, r\right]$, we get

$$
\mu(r) \geq \mu\left(r_{0}\right) e^{\int_{r_{0}}^{r} a(s) \mathrm{d} s}=\mu\left(r_{0}\right)\left(\frac{r}{r_{0}}\right)^{k_{2}-\frac{k_{1}}{|\alpha|} \varepsilon} e^{-\frac{k_{1}}{2|\alpha|}\left(r^{2}-r_{0}^{2}\right)} \quad \text { for } \quad r \geq r_{0},
$$

from which we deduce that

$$
\mu(r) \geq \frac{\mu\left(r_{0}\right)}{r_{0}-\frac{k_{1}}{|\alpha|} \varepsilon} e^{\frac{k_{1}}{2|\alpha|} r_{0}^{2}} r^{k_{2}-\frac{k_{1}}{|\alpha|} \varepsilon} e^{-\frac{k_{1}}{2|\alpha|} r^{2}} \quad \text { for } \quad r \geq r_{0} .
$$

Example 1 Another class of weight functions satisfying $\left(H_{3}\right)$, when $k_{1}=k_{2}=0$, consists of the bounded increasing functions, as, for example, $\cos e^{-|x|^{2}}$. Such a function verifies the requirements of Theorem 2 .

In the following example, we consider a wide class of functions which contains the Gaussian measure and polynomial-type measures. A class of functions which behaves as $\frac{1}{|x|^{\gamma}}$ when $|x|$ goes to zero.

Example 2 We consider the following weight functions:

$$
\mu(x)=\frac{1}{|x|^{\gamma}} e^{-\delta|x|^{m}}, \quad \delta \geq 0, \quad \gamma<N-2 .
$$

We state the values of $\gamma$ and $m$ for which the functions in (8) are "good" functions to get the weighted Hardy inequality (6).

The weight $\mu$ satisfies $\left(H_{2}\right),\left(H_{4}\right)$ and $\left(H_{5}\right)$ if $\gamma>-N$. The condition $\left(H_{3}\right)$ :

$$
\frac{\alpha\left(-\gamma-\delta m|x|^{m}\right)}{\varepsilon+|x|^{2}} \leq k_{1}+\frac{\alpha k_{2}}{\varepsilon+|x|^{2}}
$$

is fulfilled if

$$
-\left(\alpha \gamma+\alpha k_{2}+k_{1} \varepsilon\right)-\alpha \delta m|x|^{m}-k_{1}|x|^{2} \leq 0 .
$$

In the case $\delta=0$, we only need to require that $\gamma \leq-k_{2}-\frac{k_{1}}{\alpha} \varepsilon$, and we are able to get the Caffarelli-Nirenberg inequality:

$$
\left(\frac{N-2-\gamma}{2}\right)^{2} \int_{\mathbb{R}^{N}} \frac{\varphi^{2}}{|x|^{2}}|x|^{-\gamma} \mathrm{d} x \leq \int_{\mathbb{R}^{N}}|\nabla \varphi|^{2}|x|^{-\gamma} \mathrm{d} x \quad \forall \varphi \in H_{\mu}^{1} .
$$

While if $\gamma=0$, the inequality (6) holds, for $k_{1}$ large enough, with $k_{2}=0$ if $m=2$ and with $k_{2}<0$ if $m<2$.

In general to get (9), we need the following conditions on parameters and on the constant $k_{1}$ :

(i) $\quad \gamma \in\left(-N,-k_{2}\right], \delta=0, k_{1}=0$,

(ii) $\quad \gamma \in\left(-N,-k_{2}\right], k_{1} \geq-2 \alpha \delta, m=2$,

(iii) $\quad \gamma \in\left(-N,-k_{2}\right), k_{1} \geq \tilde{k}_{1}, m<2$, 
where $\tilde{k}_{1}=\frac{\frac{m}{2}\left(1-\frac{m}{2}\right)^{\frac{2}{m}-1}(-\alpha \delta m)^{\frac{2}{m}}}{\left[\alpha\left(\gamma+k_{2}\right)\right]^{\frac{2}{m}-1}}$, to get the inequality (6).

Example 3 The function $\mu(x)=[\log (1+|x|)]^{-\gamma}$, for $\gamma<N-2$, behaves as $\frac{1}{|x|^{\gamma}}$ when $|x|$ goes to 0 . So, we can state the weighted Hardy inequality (6) with $k_{1}=0$ and $\gamma \in\left(-N,-k_{2}\right]$ as in the previous example.

\section{Optimality of the constant}

To state the optimality of the constant $c_{o}\left(N+K_{2}\right)$ in the estimate (6), we need further assumptions on $\mu$ as usually it is done. We remark that in the proof of optimality, the choice of the function $\varphi$ plays a fundamental role.

We suppose

( $\left.H_{6}\right) \quad \frac{\mu(x)}{|x|^{\delta}} \in L_{\text {loc }}^{1}\left(\mathbb{R}^{N}\right)$ iff $\delta \leq N+k_{2}$.

We observe that the condition $\left(H_{6}\right)$ is necessary for the technique used to estimate the bottom of the spectrum of the operator $-L-V$ in the proof of the optimality. For example, the functions $\mu$ such that

$$
\lim _{|x| \rightarrow 0} \frac{\mu(|x|)}{|x|^{k_{2}}}=l, \quad l>0
$$

verify $\left(H_{6}\right)$.

The result below states the optimality of the constant $c_{o}\left(N+k_{2}\right)$ in the Hardy inequality.

Theorem 3 In the hypotheses $\left(H_{2}-H_{6}\right)$, the Hardy inequality (6) does not hold for any $\varphi \in H_{\mu}^{1}$ if $c>c_{o}\left(N+k_{2}\right)=\left(\frac{N+k_{2}-2}{2}\right)^{2}$.

Proof Let $\theta \in C_{c}^{\infty}\left(\mathbb{R}^{N}\right)$ be a cut-off function, $0 \leq \theta \leq 1, \theta=1$ in $B_{1}$ and $\theta=0$ in $B_{2}^{c}$. We introduce the function:

$$
\varphi_{\varepsilon}(x)= \begin{cases}(\varepsilon+|x|)^{\eta} & \text { if }|x| \in[0,1[ \\ (\varepsilon+|x|)^{\eta} \theta(x) & \text { if }|x| \in[1,2[ \\ 0 & \text { if }|x| \in[2,+\infty[,\end{cases}
$$

where $\varepsilon>0$ and the exponent $\eta$ is such that

$$
\max \left\{-\sqrt{c},-\frac{N+k_{2}}{2}\right\}<\eta<\min \left\{-\frac{N+k_{2}-2}{2}, 0\right\} .
$$

The function $\varphi_{\varepsilon}$ belongs to $H_{\mu}^{1}$ for any $\varepsilon>0$.

For this choice of $\eta$, we obtain $\eta^{2}<c,|x|^{2 \eta} \in L_{\text {loc }}^{1}\left(\mathbb{R}^{N}, \mathrm{~d} \mu\right)$ and $|x|^{2 \eta-2} \notin L_{\text {loc }}^{1}\left(\mathbb{R}^{N}, \mathrm{~d} \mu\right)$.

Let us assume that $c>c_{o}\left(N+k_{2}\right)$. In order to state the result, we prove that bottom of the spectrum of the operator $-(L+V)$ :

$$
\lambda_{1}=\inf _{\varphi \in H_{\mu}^{1} \backslash\{0\}}\left(\frac{\int_{\mathbb{R}^{N}}|\nabla \varphi|^{2} \mathrm{~d} \mu-\int_{\mathbb{R}^{N}} \frac{c}{|x|^{2}} \varphi^{2} \mathrm{~d} \mu}{\int_{\mathbb{R}^{N}} \varphi^{2} \mathrm{~d} \mu}\right),
$$

is $-\infty$. For this purpose, we estimate at first the numerator in (10) with $\varphi=\varphi_{\varepsilon}$. 


$$
\begin{aligned}
\int_{\mathbb{R}^{N}} & \left(\left|\nabla \varphi_{\varepsilon}\right|^{2}-\frac{c}{|x|^{2}} \varphi_{\varepsilon}^{2}\right) \mathrm{d} \mu \\
= & \int_{B_{1}}\left[\left|\nabla(\varepsilon+|x|)^{\eta}\right|^{2}-\frac{c}{|x|^{2}}(\varepsilon+|x|)^{2 \eta}\right] \mathrm{d} \mu \\
& +\int_{B_{1}^{c}}\left[\left|\nabla(\varepsilon+|x|)^{\eta} \theta\right|^{2}-\frac{c}{|x|^{2}}(\varepsilon+|x|)^{2 \eta} \theta^{2}\right] \mathrm{d} \mu \\
\leq & \int_{B_{1}}\left[\eta^{2}(\varepsilon+|x|)^{2 \eta-2}-\frac{c}{|x|^{2}}(\varepsilon+|x|)^{2 \eta}\right] \mathrm{d} \mu \\
& +\eta^{2} \int_{B_{1}^{c}}(\varepsilon+|x|)^{2 \eta-2} \theta^{2} \mathrm{~d} \mu+\int_{B_{1}^{c}}(\varepsilon+|x|)^{2 \eta}|\nabla \theta|^{2} \mathrm{~d} \mu \\
& +2 \eta \int_{B_{1}^{c}} \theta(\varepsilon+|x|)^{2 \eta-1} \frac{x}{|x|} \cdot \nabla \theta \mathrm{d} \mu \\
\leq & \int_{B_{1}}(\varepsilon+|x|)^{2 \eta}\left[\frac{\eta^{2}}{(\varepsilon+|x|)^{2}}-\frac{c}{|x|^{2}}\right] \mathrm{d} \mu \\
& +2 \eta^{2} \int_{B_{1}^{c}}(\varepsilon+|x|)^{2 \eta-2} \theta^{2} \mathrm{~d} \mu+2 \int_{B_{1}^{c}}(\varepsilon+|x|)^{2 \eta}|\nabla \theta|^{2} \mathrm{~d} \mu \\
\leq & \int_{B_{1}}(\varepsilon+|x|)^{2 \eta}\left[\frac{\eta^{2}}{(\varepsilon+|x|)^{2}}-\frac{c}{|x|^{2}}\right] \mathrm{d} \mu+C_{1},
\end{aligned}
$$

where $C_{1}=\left(2 \eta^{2}+2\|\nabla \theta\|_{\infty}\right) \int_{B_{1}^{c}} \mathrm{~d} \mu$.

Furthermore,

$$
\int_{\mathbb{R}^{N}} \varphi_{\varepsilon}^{2} \mathrm{~d} \mu \geq \int_{B_{2} \backslash B_{1}}(\varepsilon+|x|)^{2 \eta} \theta^{2} \mathrm{~d} \mu=C_{2, \varepsilon} .
$$

Putting together (11) and (12), we get from (10):

$$
\lambda_{1} \leq \frac{\int_{B_{1}}(\varepsilon+|x|)^{2 \eta}\left[\frac{\eta^{2}}{(\varepsilon+|x|)^{2}}-\frac{c}{|x|^{2}}\right] \mathrm{d} \mu+C_{1}}{C_{2, \varepsilon}} .
$$

Letting $\varepsilon \rightarrow 0$ in the numerator above, taking in mind that $|x|^{2 \eta} \in L_{\text {loc }}^{1}\left(\mathbb{R}^{N}, \mathrm{~d} \mu\right)$ and Fatou's lemma, we obtain:

$$
\lim _{\varepsilon \rightarrow 0} \int_{B_{1}}(\varepsilon+|x|)^{2 \eta}\left[\frac{\eta^{2}}{(\varepsilon+|x|)^{2}}-\frac{c}{|x|^{2}}\right] \mathrm{d} \mu \leq-\left(c-\eta^{2}\right) \int_{B_{1}}|x|^{2 \eta-2} \mathrm{~d} \mu=-\infty,
$$

and then, $\lambda_{1}=-\infty$.

\section{Kolmogorov operators and existence and nonexistence results}

In the standard setting, one considers $\mu \in C_{\mathrm{loc}}^{1, \lambda}\left(\mathbb{R}^{N}\right)$ for some $\lambda \in(0,1)$ and $\mu>0$ for any $x \in \mathbb{R}^{N}$.

We consider Kolmogorov operators:

$$
L u=\Delta u+\frac{\nabla \mu}{\mu} \cdot \nabla u,
$$

on smooth functions, where the probability density $\mu$ in the drift term is not necessarily $(1, \lambda)$-Hölderian in the whole space but belongs to $C_{\text {loc }}^{1, \lambda}\left(\mathbb{R}^{N} \backslash\{0\}\right)$. 
These operators arise from the bilinear form integrating by parts:

$$
a_{\mu}(u, v)=\int_{\mathbb{R}^{N}} \nabla u \cdot \nabla v \mathrm{~d} \mu=-\int_{\mathbb{R}^{N}}(L u) v \mathrm{~d} \mu .
$$

The purpose is to get existence and nonexistence results for weak solutions to the initial value problem on $L_{\mu}^{2}$ corresponding to the operator $L$ perturbed by an inverse square potential:

$$
\text { (P) }\left\{\begin{array}{l}
\partial_{t} u(x, t)=L u(x, t)+V(x) u(x, t), \quad x \in \mathbb{R}^{N}, t>0, \\
u(\cdot, 0)=u_{0} \geq 0 \in L_{\mu}^{2},
\end{array}\right.
$$

where $V(x)=\frac{c}{|x|^{2}}$, with $c>0$.

We say that $u$ is a weak solution to $(P)$ if, for each $T, R>0$, we have:

$$
u \in C\left([0, T], L_{\mu}^{2}\right), \quad V u \in L^{1}\left(B_{R} \times(0, T), \mathrm{d} \mu \mathrm{d} t\right)
$$

and

$$
\int_{0}^{T} \int_{\mathbb{R}^{N}} u\left(-\partial_{t} \phi-L \phi\right) \mathrm{d} \mu \mathrm{d} t-\int_{\mathbb{R}^{N}} u_{0} \phi(\cdot, 0) \mathrm{d} \mu=\int_{0}^{T} \int_{\mathbb{R}^{N}} V u \phi \mathrm{d} \mu \mathrm{d} t
$$

for all $\phi \in W_{2}^{2,1}\left(\mathbb{R}^{N} \times[0, T]\right)$ having compact support with $\phi(\cdot, T)=0$, where $B_{R}$ denotes the open ball of $\mathbb{R}^{N}$ of radius $R$ centered at 0 . For any $\Omega \subset \mathbb{R}^{N}, W_{2}^{2,1}(\Omega \times(0, T))$ is the parabolic Sobolev space of the functions $u \in L^{2}(\Omega \times(0, T))$ having weak space derivatives $D_{x}^{\alpha} u \in L^{2}(\Omega \times(0, T))$ for $|\alpha| \leq 2$ and weak time derivative $\partial_{t} u \in L^{2}(\Omega \times(0, T))$ equipped with the norm:

$$
\begin{aligned}
\|u\|_{W_{2}^{2,1}(\Omega \times(0, T))}:= & \left(\|u\|_{L^{2}(\Omega \times(0, T))}^{2}+\left\|\partial_{t} u\right\|_{L^{2}(\Omega \times(0, T))}^{2}\right. \\
& \left.+\sum_{1 \leq|\alpha| \leq 2}\left\|D^{\alpha} u\right\|_{L^{2}(\Omega \times(0, T))}^{2}\right)^{\frac{1}{2}} .
\end{aligned}
$$

Let us assume that the function $\mu$ is a probability density on $\mathbb{R}^{N}, \mu>0$. In the hypothesis $\left(H_{7}\right) \quad \mu \in C_{\text {loc }}^{1, \lambda}\left(\mathbb{R}^{N}\right), \lambda \in(0,1)$,

it is known that the operator $L$ with domain

$$
D_{\max }(L)=\left\{u \in C_{b}\left(\mathbb{R}^{N}\right) \cap W_{\text {loc }}^{2, p}\left(\mathbb{R}^{N}\right) \text { for all } 1<p<\infty, L u \in C_{b}\left(\mathbb{R}^{N}\right)\right\}
$$

is the weak generator of a not necessarily $C_{0}$-semigroup in $C_{b}\left(\mathbb{R}^{N}\right)$. Since $\int_{\mathbb{R}^{N}} L u \mathrm{~d} \mu=0$ for any $u \in C_{c}^{\infty}\left(\mathbb{R}^{N}\right), \mathrm{d} \mu=\mu(x) \mathrm{d} x$ is the invariant measure for this semigroup in $C_{b}\left(\mathbb{R}^{N}\right)$. So, we can extend it to a positivity preserving and analytic $C_{0}$-semigroup $\{T(t)\}_{t \geq 0}$ on $L_{\mu}^{2}$, whose generator is still denoted by $L$ (see [24]).

When the assumptions on $\mu$ allow degeneracy at one point, we require the following conditions to get that $L$ generates a semigroup:

( $\left.H_{8}\right) \quad \mu \in C_{\text {loc }}^{1, \lambda}\left(\mathbb{R}^{N} \backslash\{0\}\right), \lambda \in(0,1), \mu \in H_{\text {loc }}^{1}\left(\mathbb{R}^{N}\right), \frac{\nabla \mu}{\mu} \in L_{\text {loc }}^{r}\left(\mathbb{R}^{N}\right)$ for some $r>N$, and $\inf _{x \in K} \mu(x)>0$ for any compact set $K \subset \mathbb{R}^{N}$.

So by [1, Corollary 3.7], we have that the closure of $\left(L, C_{c}^{\infty}\left(\mathbb{R}^{N}\right)\right)$ on $L_{\mu}^{2}$ generates a strongly continuous and analytic Markov semigroup $\{T(t)\}_{t \geq 0}$ on $L_{\mu}^{2}$. 
We observe that the function $e^{-\delta|x|^{m}}$ fully satisfies the condition $\left(H_{8}\right)$ while $\cos e^{-|x|^{2}}$ is $(1, \lambda)$-Hölderian in $\mathbb{R}^{N}$ (see Examples in Sect. 2).

For weight functions $\mu$ satisfying assumption $\left(H_{7}\right)$ or $\left(H_{8}\right)$, there are some interesting properties regarding the semigroup $\{T(t)\}_{t \geq 0}$ generated by the operator $L$. These properties listed in the Proposition below are well known under hypothesis $\left(H_{7}\right)$ (see [24]) and have been proved in [9] if $\mu$ satisfies $\left(H_{8}\right)$.

Proposition 1 Assume that $\mu$ satisfies $\left(\mathrm{H}_{7}\right)$ or $\left(\mathrm{H}_{8}\right)$. Then, the following assertions hold:

(i) $D(L) \subset H_{\mu}^{1}$.

(ii) For every $f \in D(L), g \in H_{\mu}^{1}$ we have:

$$
\int L f g \mathrm{~d} \mu=-\int \nabla f \cdot \nabla g \mathrm{~d} \mu .
$$

(iii) $T(t) L_{\mu}^{2} \subset D(L)$ for all $t>0$.

The following Theorem stated in [20] for functions $\mu$ satisfying condition $\left(\mathrm{H}_{7}\right)$ was proved in [9] for functions $\mu$ under condition $\left(H_{8}\right)$.

Theorem 4 Let $0 \leq V(x) \in L_{\text {loc }}^{1}\left(\mathbb{R}^{N}\right)$. Assume that the weight function $\mu$ satisfies $\left.H_{4}\right)$, $\left.H_{5}\right)$ and $\left.\mathrm{H}_{8}\right)$. Then, the following assertions hold:

(i) If $\lambda_{1}(L+V)>-\infty$, then there exists a positive weak solution $u \in C\left([0, \infty), L_{\mu}^{2}\right)$ of $(P)$ satisfying

$$
\|u(t)\|_{L_{\mu}^{2}} \leq M e^{\omega t}\left\|u_{0}\right\|_{L_{\mu}^{2}}, \quad t \geq 0
$$

for some constants $M \geq 1$ and $\omega \in \mathbb{R}$.

(ii) If $\lambda_{1}(L+V)=-\infty$, then for any $0 \leq u_{0} \in L_{\mu}^{2} \backslash\{0\}$, there is no positive weak solution of $(P)$ satisfying (14).

To get existence and nonexistence of solutions to $(P)$, we put together the weighted Hardy inequality (2), Theorems 3 and 4 . So, we can state the following result.

Theorem 5 Assume that the weight function $\mu$ satisfies hypotheses $\left(H_{2}-H_{6}\right),\left(H_{8}\right)$ and $0 \leq$ $V(x) \leq \frac{c}{|x|^{2}}$. The following assertions hold:

(i) If $0 \leq c \leq c_{o}\left(N+k_{2}\right)=\left(\frac{N+k_{2}-2}{2}\right)^{2}$, then there exists a positive weak solution $u \in C\left([0, \infty), L_{\mu}^{2}\right)$ of $(P)$ satisfying

$$
\|u(t)\|_{L_{\mu}^{2}} \leq M e^{\omega t}\left\|u_{0}\right\|_{L_{\mu}^{2}}, \quad t \geq 0
$$

for some constants $M \geq 1, \omega \in \mathbb{R}$, and any $u_{0} \in L_{\mu}^{2}$.

(ii) If $c>c_{o}\left(N+k_{2}\right)$, then for any $0 \leq u_{0} \in L_{\mu}^{2}, u_{0} \neq 0$, there is no positive weak solution of $(P)$ with $V(x)=\frac{c}{|x|^{2}}$ satisfying (15).

\section{References}

1. Albanese, A., Lorenzi, L., Mangino, E.: $L^{p}$-uniqueness for elliptic operators with unbounded coefficients in $\mathbb{R}^{N}$. J. Funct. Anal. 256, 1238-1257 (2009)

2. Aronson, D.G.: Non-negative solutions of linear parabolic equations. Ann. Sc. Norm. Super. Pisa 22, 607-694 (1968) 
3. Baras, P., Goldstein, J.A.: The heat equation with a singular potential. Trans. Am. Math. Soc. 284, 121-139 (1984)

4. Baras, P., Goldstein J.A.: Remarks on the inverse square potential in quantum mechanics. In: Knowles, I., Lewis, R. (eds.) International Conference on Differential Equations, North-Holland, Amsterdam (1984)

5. Berestycki, H., Esteban, M.J.: Existence and bifurcation of solutions for an elliptic degenerate problem. J. Differ. Equ. 134(1), 1-25 (1997)

6. Besov, O.V.: On the denseness of compactly supported functions in a weighted Sobolev space. Trudy Mat. Inst. Steklov. 161, 29-47 (1983). English transl. in Proc. Mat. Inst. Steklov 161 (1984)

7. Bogachev, V.I.: Differentiable Measures and the Malliavin Calculus. Mathematical Surveys and Monographs 164. American Mathematical Society, Providence (2010)

8. Cabré, X., Martel, Y.: Existence versus explosion instantanée pour des équations de la chaleur linéaires avec potentiel singulier. C. R. Acad. Sci. Paris 329(11), 973-978 (1999)

9. Canale, A., Gregorio, F., Rhandi, A., Tacelli, C.: Weighted Hardy's inequalities and Kolmogorov-type operators. Appl. Anal., pp. 1-19 (2017)

10. Canale, A., Mininni, R.M., Rhandi, A.: Analytic approach to solve a degenerate parabolic PDE for the Heston model. Math. Meth. Appl. Sci. 40, 4982-4992 (2017)

11. Canale, A., Pappalardo, F.: Weighted Hardy inequalities and Ornstein-Uhlenbeck type operators perturbed by multipolar inverse square potentials. J. Math. Anal. Appl. 463, 895-909 (2018)

12. Canale, A., Pappalardo, F., Tarantino, C.: Weighted multipolar Hardy inequalities and evolution problems with Kolmogorov operators perturbed by singular potentials, arXiv:1908.01971v1, 6 Aug (2019)

13. Canale, A., Rhandi, A., Tacelli, C.: Schrödinger type operators with unbounded diffusion and potential terms. Ann. Sc. Norm. Super. Pisa Cl. Sci. 16, 581-601 (2016)

14. Canale, A., Rhandi, A., Tacelli, C.: Kernel estimates for Schrödinger type operators with unbounded diffusion and potential terms. Z. Anal. Anwend. 36, 377-392 (2016)

15. Canale, A., Tacelli, C.: Kernel estimates for a Schrödinger type operator. Riv. Mat. Univ. Parma 7, 341-350 (2016)

16. Chiadó Piat, V., Serra Cassano, F.: Some remarks about the density of smooth functions in weighted Sobolev spaces. J. Convex Anal. 1(2), 135-142 (1994)

17. Davies, E.B.: Spectral Theory and Differential Operators, Cambridge Studies in Advanced Mathematics 42. Cambridge University Press, Cambridge (1995)

18. Fabes, E.B., Kenig, C.E., Serapioni, R.P.: The local regularity of solutions of degenerate elliptic equations. Comm. Partial Differ. Equ. 7, 77-116 (1982)

19. Gel'fand, I.M.: Some problems in the theory of quasi-linear equations. Uspehi Mat. Nauk 14, 87-158 (1959)

20. Goldstein, G.R., Goldstein, J.A., Rhandi, A.: Weighted Hardy's inequality and the Kolmogorov equation perturbed by an inverse-square potential. Appl. Anal. 91(11), 2057-2071 (2012)

21. Kufner, A.: Weighted Sobolev spaces, Texte zur Mathematik 31. Teubner, Stuttgart-Leipzig-Wiesbaden (1980)

22. Kufner, A., Opic, B.: How to define reasonably weighted Sobolev spaces. Comment. Math. Univ. Carolinae 25(3), 537-554 (1984)

23. Lévy-Leblond, J.M.: Electron capture by polar molecules. Phys. Rev. 153(1), 1-4 (1967)

24. Lorenzi, L., Bertoldi, M.: Analytical Methods for Markov Semigroups, Pure and Applied Mathematics 283. CRC Press, Boca Raton (2006)

25. Tölle, J.M.: Uniqueness of weighted Sobolev spaces with weakly differentiable weights. J. Funct. Anal. 263, 3195-3223 (2012)

26. Zhikov, V.V.: Weighted Sobolev spaces. Sb. Math. 189(8), 1139-1170 (1998). Translated from Math. Sb. 189(8), 27-58 (1998)

Publisher's Note Springer Nature remains neutral with regard to jurisdictional claims in published maps and institutional affiliations. 